\title{
VIEWPOINT
}

\section{Modeling the course of Alzheimer's disease to improve clinical trials: symposium report}

\author{
René Spiege ${ }^{* 1}$, Rachelle S Doody ${ }^{2}$, Suzanne Hendrix ${ }^{3}$ and Kristin Kahle-Wrobleski ${ }^{4}$
}

\begin{abstract}
In a symposium held at the Clinical Trials in Alzheimer's Disease conference in Monte Carlo, Monaco (29 to

31 October 2012) three different, not mutually exclusive approaches to improve and facilitate clinical trials with anti-dementia drugs were presented and discussed. All three approaches are summarized in this manuscript. Core suggestions are: stratification of trial participants at the outset of studies, using cognitive and disease-course characteristics available at baseline; creating new composite cognitive scores for optimizing responsiveness to decline in early and very early Alzheimer's disease; and replacing some of the conventional long-term placebo-controlled trials in advanced stages of drug development, using the placebo group simulation approach. Future efforts should focus on incorporating, where appropriate, the suggestions provided at the symposium into clinical trials now being planned.
\end{abstract}

\section{Introduction}

Despite existing treatments and recent advances in potential targets for pharmacological interventions, there remains a great unmet need in the treatment of Alzheimer's disease (AD). Progress toward new therapeutic agents is hampered by difficulties in the related issues of measurement of disease/symptom pathology and prediction of disease course, both of which impact many facets of drug development including study design, power and sample size calculations, and interpretability of results. Difficulties in measurement and prediction are even more pronounced when moving into a milder or presymptomatic patient population.

A symposium held at the Clinical Trials in Alzheimer's Disease conference in Monte Carlo, Monaco (29 to

*Correspondence: rene.spiegel@unibas.ch

'Department of Geriatrics, Memory Clinic, University Hospital, CH-4031 Basel, Switzerland

Full list of author information is available at the end of the article
31 October 2012) dealt with some of these issues, emphasizing the potential of different modeling approaches. Modeling the course of $\mathrm{AD}$ at different stages of its development, from the presymptomatic stages to severe dementia, is assumed to contribute to better planning and design of future clinical trials. Three different approaches using patient populations from across the diagnostic spectrum of the disease - probable/possible $\mathrm{AD}$, mild cognitive impairment (MCI) and amnestic MCI - were presented and are summarized in the following paragraphs.

\section{Predicting progression of Alzheimer's disease: understanding the variance in progression of $A D$ (Rachelle Doody)}

In their pioneering work, Rachelle Doody and colleagues assert that modeling of group disease progression is critical for estimating change in clinical trials of diseasemodifying therapies. Despite years of observation in small studies, there is no clear model for how AD progresses or for the sources of variance in progression between patients. According to these authors, multivariate models of disease progression can also identify the factors that contribute to the variance and thereby foster the ability to stratify patients in clinical trials or to predict progression in individual patients. Doody and her group have shown that an estimate of early disease progression, the pre-progression rate, is predictive of the time it will take to develop substantial decline [1].

Doody and her group have also used mixed-effects regression analysis to examine the role of demographic, biological, clinical and psychometric characteristics in predicting progression of $\mathrm{AD}$ in a large patient cohort [2]. This work suggests that premorbid IQ (probably a surrogate for cognitive reserve) and the early, intrinsic progression rate are the most predictive variables, yet these are seldom captured as baseline characteristics and are therefore seldom balanced or accounted for in the analysis of clinical trials. The persistence of anti-dementia drug use from the time of symptom onset is also a predictive variable [3], whereas most study protocols simply collect information on concurrent drug use at the time of randomization. Although the pre-progression 
rate and premorbid IQ could also be used as fixed effects or covariates in analysis of clinical trials data, stratification is preferred in order to ensure that there are equal-sized groups of those above and below median IQ (reflecting cognitive reserve), and equal-sized groups of slow, intermediate and rapidly progressing patients (possibly reflecting biological differences) since treatment response could conceivably differ between these groups.

Although the Doody group has been modeling the progression rate for some time, the question of interindividual variance in progression rates is a new focus of investigation. The new analysis suggests that the factors contributing most to variance in outcomes - such as the Alzheimer Disease Assessment Scale cognitive (ADAScog), Clinical Dementia Scale Sum of Boxes (CDR-SB), Instrumental Activities of Daily Life, Physical SelfMaintenance Scale, and Mini Mental State Examination (MMSE) - are the pre-progression rate, the presence or development of parkinsonism, and the presence or development of psychosis. In their multivariable model, which includes time, the American National Adult Reading Test estimate of IQ, the progression rate and the presence or development of parkinsonism or psychosis, one-third to one-half of variance between individuals was explained, depending on which outcome (MMSE, ADAS, CDR-SB, Physical Self-Maintenance Scale, Instrumental Activities of Daily Life) was considered.

Taken together, the studies on progression rates and variance suggest that patients should be stratified based upon IQ and pre-progression rate (as opposed to MMSE), and that studies should capture the development of parkinsonism or psychosis for use as time-dependent covariates in the comparison of treatment groups. Studies should probably also quantify disease-long exposure to anti-dementia drugs, as opposed to simply recording their use during the clinical trial. Although the potential for enriching clinical trials populations through the use of IQ and pre-progression rates also exists, the authors would not recommend this until their effects in treatment trials are better understood.

\section{Creating a new composite score for optimizing responsiveness to decline in early $A D$ and very early AD (Suzanne Hendrix)}

Suzanne Hendrix has proposed empirically derived composite outcome scores that optimize the power for measuring clinical disease progression for trials in $\mathrm{MCI}$ and pre-MCI populations [4]. The goal of this work is to improve the responsiveness of clinical outcomes to disease progression in early stages of $\mathrm{AD}$, with the expectation that this will give treatments the best chance for showing an effect. The proposed composite outcome scores for MCI utilize items from standard AD instruments, such as the ADAS-cog, but rather than summing all item scores they use an optimization algorithm to select and weigh the items that are declining most in the population of interest, excluding items that are not yet declining in these early $\mathrm{AD}$ populations.

Approaches based on principal components factor analysis and different regression techniques have been compared for robustness over multiple study datasets and across enriched (amyloid beta 42-positive, apolipoprotein E4 allele carriers) and non-enriched populations. When ADAS-cog, MMSE and CDR-SB items are considered, similar item combinations are identified as responsive to disease progression despite the diversity of the populations and methodology, indicating that the majority of MCI subjects in clinical studies have a common symptom profile over time. The items that are common across populations and methodologies are delayed word recall and orientation from the ADAS-cog, orientation to time from the MMSE and all six CDR box scores. The partial least-squares model - which is a compromise between an item response theory or principal components approach and an approach that directly optimizes the sensitivity to changes over time appears to result in the most robust combinations, and excludes redundant items with lower responsiveness from the composite score. This method also estimates the optimal weighting of the items to reflect the relative importance of each item in the population under consideration.

Composite scores can be constructed utilizing cognitive items only, global or functional items only or a combination for optimal responsiveness. This methodology could also be applied to biomarker data in order to identify which biomarkers measure decline that is not redundant with clinical outcomes. These responsive composite clinical outcomes allow smaller and shorter clinical trials in early disease, and will help with validation of biomarkers in these early stages. Population enrichment provides additional improvement over item optimization alone [5], but optimizing within an enriched population does not result in substantially different item combinations, indicating that the methodology successfully identifies the disease-specific decline across homogeneous or heterogeneous MCI patient populations.

\section{The placebo group simulation approach: an alternative to long-term placebo-controlled trials (René Spiegel)}

René Spiegel presented his teams' development work on the placebo group simulation approach (PGSA), a novel clinical study design for use in long-term trials with putative disease-course altering drugs for use in $\mathrm{AD}$. The PGSA is intended to circumvent a major limitation of randomized placebo-controlled double-blind clinical trial designs; that is, the need to expose prodromal $\mathrm{AD}$ 
patients at high risk for dementia to extended placebo treatment, which may result in problems with patient recruitment [6], questions about the representativeness of study samples and ethical issues.

The PGSA uses stochastic modeling to forecast predefined endpoints and trajectories of neuropsychological outcomes from the data that are routinely available at the outset of clinical trials: basic demographic, biological and neuropsychological data for all study participants. These model-based, forecasted endpoints and trajectories of the study sample constitute the background - the simulated placebo group - against which potential drug effects can be contrasted.

Development and initial testing of PGSA algorithms for the ADAS-cog and the composite score of a neuropsychological test battery (NP-Batt9) using data from the Alzheimer's Disease Neuroimaging Initiative (ADNI 1) MCI patient cohort [7] were described in Spiegel and colleagues [8]. A new analysis of data from the ADNI 1 $\mathrm{AD}$ patient cohort, using the published PGSA algorithm for the NP-Batt9, confirms the high concordance between the model-based forecasted data and the actually observed neuropsychological data (Figure 1) and supports the application of the PGSA algorithm for the neuropsychological test battery used in ADNI 1 over a wide spectrum of preclinical and clinical AD patients [9].

The PGSA algorithm developed from the ADNI 1 MCI database was then adapted to a modified neuropsychological battery and applied to data from amnestic MCI patients available in the National Alzheimer's Coordination Center database (grant number U01 AG016976). The results obtained from this larger and less narrowly defined patient sample confirm that mathematically modeled trajectories of neuropsychological measures show high concordance with the empirically observed outcomes in amnestic MCI patients. Based on these retrospective analyses of publicly available data from large $\mathrm{MCI}$ and $\mathrm{AD}$ patient samples, Spiegel and colleagues thus conclude that the PGSA algorithms for the ADAS-cog and a NP-Batt allow valid forecasts of the distribution of the trajectories and endpoints of relevant neurocognitive outcomes recorded from amnestic MCI and symptomatic AD patients. Pending further confirmatory studies in international sets of data, prospective studies should be performed, preferably in the context of real-life anti-AD drug development. Given their inherent limitations (focus on quantified indices of efficacy, lack of concomitant controls for potential drug side effects), PGSA-based trials are expected to complement randomized placebo-controlled trials in the later phases of anti-AD drug development; that is, at stages when there is adequate evidence of a drug's efficacy and safety available from preceding placebo-controlled clinical studies.

\section{Discussion (Kristin Kahle-Wrobleski)}

The difficulties of measurement and prediction in $\mathrm{AD}$ are not easily fixed given the inherent complexities of the disease state. These complexities notwithstanding, consensus within the scientific community on favorable approaches for modeling and predicting disease course is necessary to help regulatory and payor agencies make informed decisions about the approvability and accessibility of the next generation of pharmacological interventions. The approaches listed are important anchor points for building this consensus and provide relatively straightforward suggestions for improving measurement and prediction in $\mathrm{AD}$ : control for as many known variables as possible; use the most sensitive items/scales possible; and use modeling whenever possible to minimize the number of patients needed in trials.

Doody and colleagues draw on an extensive literature as well as their own institutional research to suggest a set of measures that are easily adapted for use in clinical trials, including premorbid IQ and persistence of treatment with cholinesterase inhibitors/memantine (not just a binary variable of use). Furthermore, the time since onset of symptoms can help model individual trajectories of progression to inform models. Hendrix similarly looks at time as a crucial variable to inform how best to maximize the sensitivity of current scales. With this approach, scale items are retained in a composite only if those items are strongly related to progression over time. Existing scales can therefore be combined to move away from thinking of scales as tools of staging or description, towards using scales as more powerful and sensitive instruments that can shorten trial times and reduce the number of patients needed to enroll in trials. Spiegel and colleagues also sought to find ways to minimize the number of patients needed in trials and exposed to longterm placebo treatment. Their approach, like Doody and colleagues and Hendrix and colleagues, uses existing scales to build a simulated placebo group that could be used instead of an actual placebo group for trials in advanced stages of drug development.

As with most modeling approaches, additional validation is needed in a variety of datasets before a gold standard is likely to be declared. Most of the datasets used to inform these approaches come from highly specialized centers that see patients who are unlikely to represent the broader patient population. Conceptually, these approaches should apply to international patient populations, and additional validation work with datasets from various countries (for example, European Alzheimer's Disease Consortium, Australian Imaging, Biomarker, and Lifestyle Flagship Study of Ageing) might provide evidence of generalizability. Other beneficial validation work may look at what, if any, differences exist in model fit when differing diagnostic criteria [10-12] are 


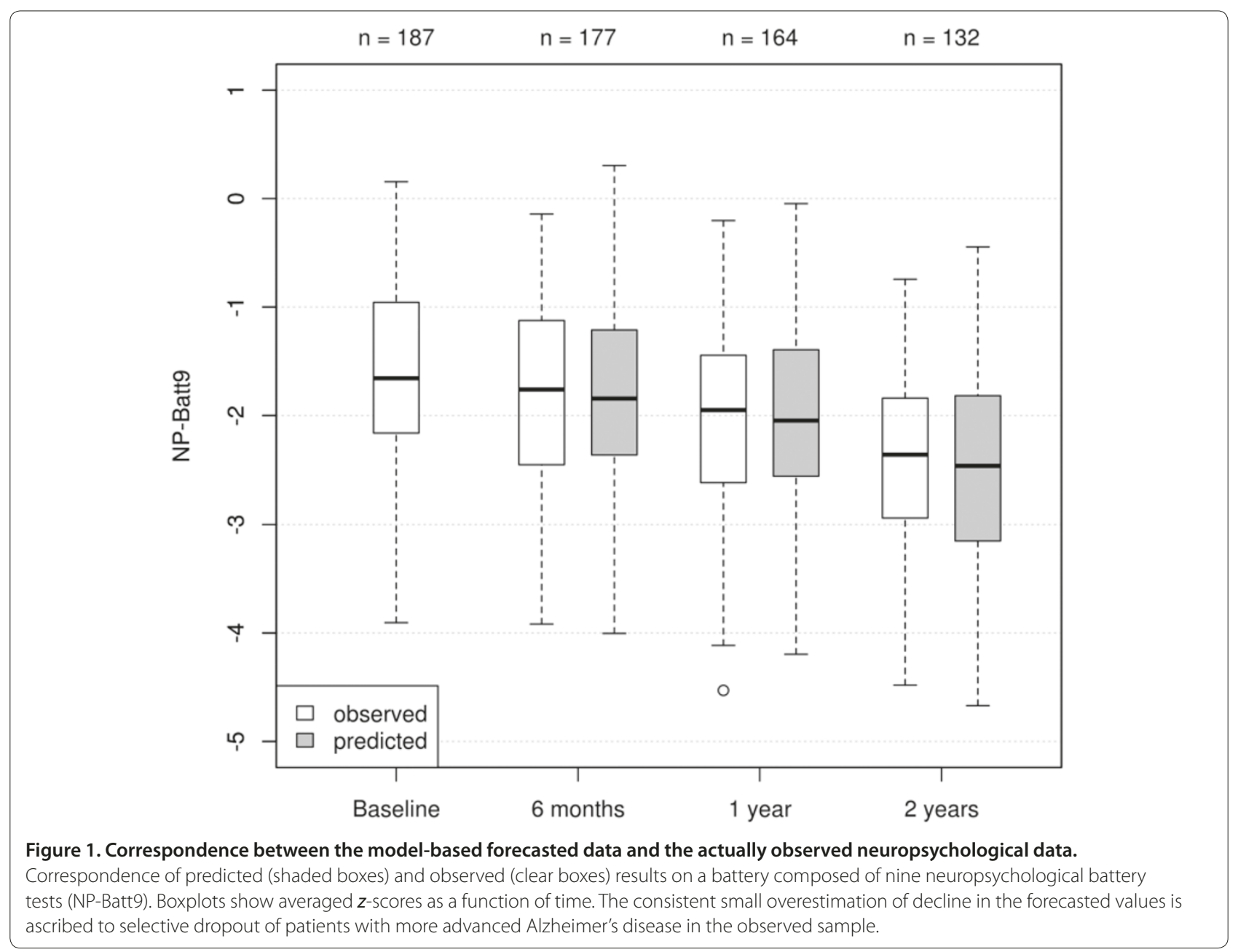

used to select patient cohorts. Further, as biomarkers evolve and the field becomes better able to distinguish underlying neuropathologies, models of clinical symptoms are likely to need further refinement. Another key consideration when evaluating the impact of these modeling approaches is that the purpose of clinical trials is not just to show benefit of a drug; the trials should also characterize and quantify the risks associated with the therapy. Discussions with regulators and payors will therefore be needed to help industry sponsors understand how best to collect safety data within the context of shorter studies or potentially, as Spiegel and colleagues suggest, in the absence of a long-term placebo group that might inform base rates of events.

Although alternative approaches using new scales have been suggested as a way of improving the speed and accuracy of trials (for example [13]), the modeling approaches described here make use of scales widely used in clinical trials, patient registries and observational studies. The advantage of using existing scales versus creating new ones is not inconsequential. These approaches more easily allow validation with complimentary datasets and also facilitate comparisons between the approaches. In discussions with regulatory and payor agencies, it is also helpful to include measures with a substantial research history across a variety of settings so that discussions can productively focus on assessing benefits and risks rather than debating measurement methodology. Modeling approaches such as the ones described here help provide stakeholders with pragmatic solutions to running trials that may be fairly easy to implement and provide additional rigor for detecting drug effects in a more timely manner.

\section{Conclusion}

Current efforts in the modeling of disease progression have grown increasingly sophisticated in an effort to account for the heterogeneity of patients across the spectrum of AD. Accurately predicting the rate of progression is essential to the drug development process, as it is likely to facilitate detecting a signal in clinical trials and to reduce uncertainty in extrapolating treatment 
effects beyond the trial time frame. Future efforts should focus on incorporating, where appropriate, the suggestions provided in the symposium into clinical trials now being planned and on furthering discussions with regulatory and payor agencies as to the acceptability and interpretability of the proposed modeling approaches.

\section{Abbreviations}

AD, Alzheimer's disease; ADAS-cog, Alzheimer Disease Assessment Scale cognitive; ADNI, Alzheimer's Disease Neuroimaging Initiative; CDR-SB, Clinical Dementia Scale Sum of Boxes; IQ, intelligence quotient; $\mathrm{MCl}$, mild cognitive impairment; MMSE, Mini Mental State Examination; NP-Batt, Neuropsychological Battery; PGSA, placebo group simulation approach.

\section{Competing interests}

$\mathrm{SH}$ is the president of Pentara Corporation, a company that provides services to sponsors of clinical trials, and has provided consultation on optimizing study outcomes for Eisai, Roche and the Alzheimer's Prevention Initiative. RS, together with Manfred Berres, André R Miserez and Andreas U Monsch, owns Plasima $\mathrm{GmbH}$, a small business that provides services to sponsors of clinical trials, including application of the PGSA discussed in this paper. KK-W is an employee of Eli Lilly and Company. RSD declares that she has no competing interests.

\section{Acknowledgments}

RSD is supported by the Cain Foundation. RS acknowledges the contributions made by his colleagues Manfred Berres, André R Miserez and Andreas U

Monsch in the development of the PGSA.

\section{Author details}

'Department of Geriatrics, Memory Clinic, University Hospital, CH-4031 Basel, Switzerland. ${ }^{2}$ Alzheimer's Disease and Memory Disorders Center, Department of Neurology, Baylor College of Medicine, 1977 Butler Blvd, Suite E5.101, Houston, TX 77030, USA. ${ }^{3}$ Pentara Corporation, 2180 East Claybourne Avenue, Salt Lake City, UT 84109, USA. ${ }^{4}$ Global Health Outcomes, Eli Lilly \& Company, Indianapolis, IN 46285, USA.

Published: 17 June 2013

\section{References}

1. Doody, RS, Massman, P, Dunn JK: A method for estimating progression rates in Alzheimer's disease. Arch Neurol 2001, 58:449-454.

2. Doody R, PavlikV, Massman P, Rountree S, Darby E, Chan W: Predicting progression of Alzheimer's disease. Alzheimers Res Ther 2010, 2:2. doi: 10.1186/alzrt25.
3. Rountree SD, Chan W, Pavlik V, Darby E, Siddiqui S, Doody R: Persistent treatment with cholinesterase inhibitors and memantine slows clinical progression of Alzheimer's disease (AD). Alzheimers Res Ther 2009, 1:7. doi: $10.1186 /$ alzrt7.

4. Hendrix SB: Measuring clinical progression in $\mathrm{MCl}$ and pre-MCI populations: enrichment and optimizing clinical outcomes over time. Alzheimers Res Ther 2012, 4:24.

5. Hendrix SB: Requiring an amyloid- $\beta 1-42$ biomarker may improve the efficiency of a study, and simulations may help in planning studies. Alzheimers Res Ther 2011, 3:10.

6. Grill JD, Karlawish J: Addressing the challenges to successful recruitment and retention in Alzheimer's disease clinical trials. Alzheimers Res Ther 2010, 2:34-44.

7. Alzheimer's Disease Neuroimaging Initiative [www.loni.ucla.edu/ADNI]

8. Spiegel R, Berres M, Miserez AR, Monsch AU: For debate: substituting placebo controls in long-term Alzheimer's prevention trials. Alzheimers Res Ther 2011, 3:9-20.

9. Spiegel $\mathrm{R}$, Berres $\mathrm{M}$, Monsch $\mathrm{AU}$ : The placebo group simulation approach (PGSA): its validity when applied to data from Alzheimer patients in the ADNI 1database [poster abstract]. J Nutr Health Aging 2012, 16:828,

10. Dubois B, Feldman HH, Jacova C, Dekosky ST, Barberger-Gateau P, Cummings J, Delacourte A, Galasko D, Gauthier S, Jicha G, Meguro K, O'Brien J, Pasquier F, Robert Ph, Rossor M, Salloway S, Stern Y, Visser PJ, Scheltens Ph: Research criteria for the diagnosis of Alzheimer's disease: revising the NINCDSADRDA criteria. Lancet Neurol 2007, 6:734-746.

11. Albert MS, DeKosky ST, Dickson D, Dubois B, Feldman HH, Fox NC, Gamst A, Holtzman DM, Jagust WJ, Petersen RC, Snyder PJ, Carrillo MC, Thies B, Phelps $\mathrm{CH}$ : The diagnosis of mild cognitive impairment due to Alzheimer's disease: recommendations from the National Institute on AgingAlzheimer's Association workgroups on diagnostic guidelines for Alzheimer's disease. Alzheimers Dement 2011, 7:270-279.

12. McKhann GM, Knopman DS, Chertkow H, Hyman BT, Jack CR, Kawas CH, Klunk WE, Koroshetz WJ, Manly JJ, Mayeux R, Mohs RC, Morris JC, Rossor MN, Scheltens PH, Carrillo MC, Thies B, Weintraub S, Phelps CH: The diagnosis of dementia due to Alzheimer's disease: recommendations from the National Institute on Aging-Alzheimer's Association workgroups on diagnostic guidelines for Alzheimer's disease. Alzheimers Dement 2011 7:263-269.

13. Harrison J, Minassian SL, Jenkins L, Black RS. The NTB: a neuropsychological test battery for use in Alzheimer's disease clinical trials. Arch Neurol 2007, 64:1323-1329.

\section{doi:10.1186/alzrt183}

Cite this article as: Spiegel R, et al:: Modeling the course of Alzheimer's disease to improve clinical trials: symposium report. Alzheimer's Research \& Therapy 2013, 5:29. 